THE effect of PAF on the plasma membrane polarity of polymorphonuclear leukocytes (PMNs) was investigated by measuring the steady-state fluorescence emission spectra of 2-dimethylamino(6-lauroyl) naphthalene (Laurdan), which is known to be incorporated at the hydrophobic-hydrophilic interface of the bilayer, displaying spectral sensitivity to the polarity of its surrounding. Laurdan shows a marked steady-state emission blue-shift in non-polar solvents, with respect to polar solvents. Our results demonstrate that PAF $\left(10^{-7} \mathrm{M}\right)$ induces a blue shift of the fluorescence emission spectra of Laurdan. These changes are blocked in the presence of the PAF antagonist, L-659,989. Our data indicate that the interaction between PAF and PMNs is accompanied by a decrease in polarity in the hydrophobic-hydrophilic interface of the plasma membrane.

Key words: Laurdan, PAF, Plasma membrane, Polymorphonuclear leukocytes

\section{Effect of PAF on polymorphonuclear leucocyte plasma membrane polarity: a fluorescence study}

\author{
A. Kantar, ${ }^{1, C A}$ P. L. Giorgi, ${ }^{1}$ and R. Fiorini ${ }^{2}$
}

Departments of ${ }^{1}$ Pediatrics and ${ }^{2}$ Biochemistry, University of Ancona, via Corridoni 11, 60123 Ancona, Italy

${ }^{\mathrm{CA}}$ Corresponding Author

\section{Introduction}

Platelet-activating factor (PAF, 1-O-alkyl-2-Oacetyl-sn-glyceryl-3-phosphocholine) is a potent lipid mediator with a diverse spectrum of biological activities in a variety of cells. ${ }^{1,2}$ These activities are due to the interaction of PAF with its receptors. ${ }^{3}$ Human polymorphonuclear leukocytes (PMNs) plasma membrane possess a cell surface receptor that specifically binds PAF and initiates various biochemical responses to PAF. ${ }^{3,4}$ Several studies have provided information on the nature of the interactions of PAF with PMNs membrane receptor and the signalling pathways involved in its cellular action. $^{3-5}$

Fluorescence spectroscopy techniques have been widely used to study the physico-chemical changes of membrane organization. ${ }^{6-8}$ The fluorescent membrane probe Laurdan (2-dimethylamino(6lauroyl) naphthalene) is known to be sensitive to the polarity of the environment, displaying spectral sensitivity to the phospholipid phase state.' In the phospholipid's liquid-crystalline phase and within the phase transition, Laurdan displays a red-shifted spectrum. The red shift has been attributed to relaxation processes that involve motions of the phospholipid polar head groups when they undergo reorientation around the excited state of the probe. On the other hand, in the gel phase the decrease of motional freedom of the bilayer polar residues results in an unrelaxed blue-shift spectrum.?

In this study we have investigated the effect of PAF on membrane polarity of PMNs by measuring steady-state emission spectra of Laurdan incorporated into the PMNs plasma membrane before and after addition of PAF in the absence or presence of the PAF antagonist ${ }^{10}( \pm)$-trans-2-(3-methoxy-5methylsulphonyl - 4 - propoxyphenyl) - 5 - (3,4,5 - tri methyloxyphenyl) tetrahydrofuran (L-659,989).

\section{Materials and Methods}

Preparation of PMNs: Human PMNs were isolated from freshly drawn blood of ten healthy volunteers using a Mono-Poly Resolving Medium (ICN Biomedicals, Milan, Italy) as previously described. ${ }^{11}$ Cells were suspended in Krebs-Ringer phosphate solution supplemented with $1 \mathrm{mg} / \mathrm{ml}$ glucose at a final concentration of $10^{6} \mathrm{PMNs} / \mathrm{ml}$ for chemiluminescence and fluorescence studies.

Chemiluminescence measurements: Luminol amplified chemiluminescence was measured in an AutoLumat LB 953 (Berthold Co, Wilbad, Germany) and PMNs (in the absence or presence of Laurdan) were activated by addition of PAF (Sigma Chemical Co., St Louis, MO, USA) $\left(5 \times 10^{-8} \mathrm{M}\right)$ as previously described. ${ }^{12}$

Fluorescence measurements: PMNs were labelled with an ethanol solution of Laurdan (Molecular Probes Inc. Eugene, OR, USA) under $\mathrm{N}_{2}$ at a final probe concentration of $0.05 \mu \mathrm{M}$ as previously described. ${ }^{13}$ Samples were prepared at room temperature in red light and used immediately for fluorescence measurements. Steady-state excitation and emission spectra were measured at $37^{\circ} \mathrm{C}$ before and after addition of PAF on a photon counting spectrofluorometer (model GREG PC, ISS Inc., Urbana, IL, USA) and using the ISS Inc. software. The actual temperature was measured in the sample cuvette using a digital thermometer. PAF was 
added to PMNs at a final concentration of $10^{-7} \mathrm{M}$. The PAF antagonist, L-659,989 (kindly donated by Dr W. H. Parsons, Merck Sharp \& Dohme Research Laboratories, NJ, USA) was added to PMNs at a final concentration of $10^{-6} \mathrm{M}^{10}$

\section{Results}

Chemiluminescence studies: Luminol amplified chemiluminescence has been employed, as previously described, ${ }^{12,13}$ to verify that in isolated PMNs the superoxide-generating oxidase system is dormant under basal conditions and can be activated by PAF. All samples, used in this study, demonstrated an activable NADPH-oxidase system (data not shown). PMNs labelled with Laurdan $0.05 \mu \mathrm{M}$ still showed a normal oxidative burst.

Fluorescence studies: The background phospholipid fluorescence of PMNs was checked prior to each measurement and was less than $0.1 \%$ of the fluorescence when Laurdan was added. The contribution of the light scattering was negligible in our samples because of the low cell concentration used in this study. The fluorescence intensity of the free probe in KRP, in the absence of PMNs, was negligible and did not increase upon the addition of PAF at the concentrations used in the study; moreover, this concentration $\left(10^{-7} \mathrm{M}\right)$ is lower than the critical micelle concentration of PAF $2 \times 10^{-5} \mathrm{M}$ as reported in literature. ${ }^{14}$ The kinetic of incorporation of Laurdan $(0.05 \mu \mathrm{M})$ in PMNs plasma membranes was established as recently described. ${ }^{13}$

Fluorescence emission and excitation spectra of Laurdan in PMNs before and after addition of PAF $10^{-7} \mathrm{M}$ are reported in Figs 1 and 2, respectively. Figure 1 shows emission spectra measured at an excitation wavelength of $350 \mathrm{~nm}$. After the addition

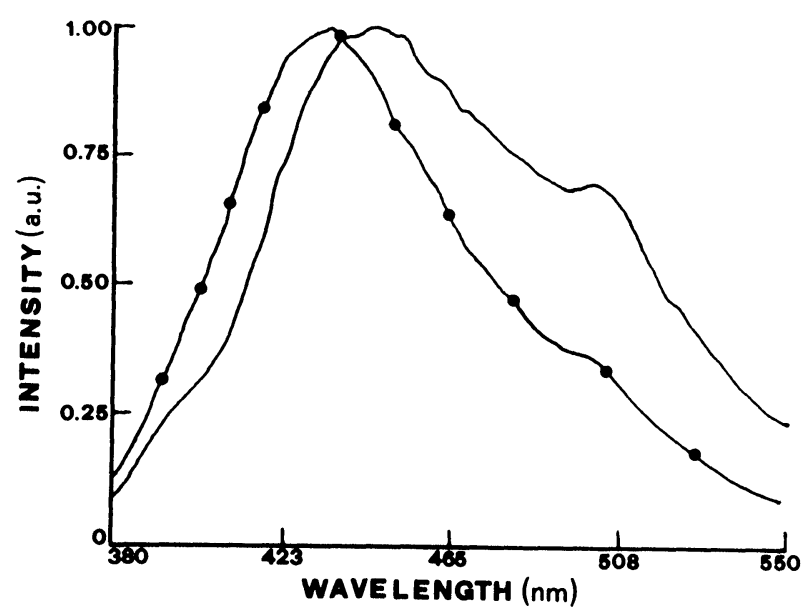

FIG. 1. Normalized emission spectra of Laurdan incorporated in PMNs plasma membrane at $37^{\circ} \mathrm{C}$ before (-) and after (- - ) addition of PAF $10^{-7} \mathrm{M}$. The spectra were measured at an excitation wavelength of $350 \mathrm{~nm}$.

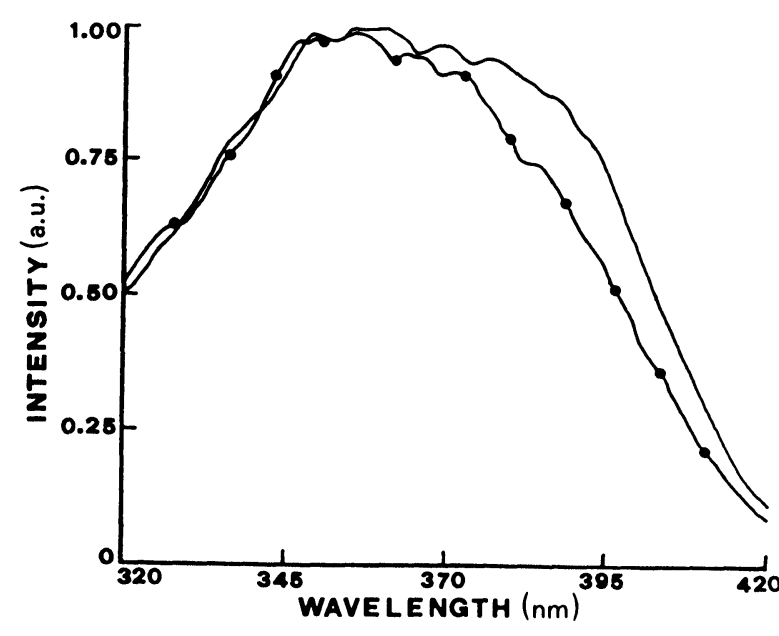

FIG. 2. Normalized excitation spectra of Laurdan incorporated in PMNs plasma membrane at $37^{\circ} \mathrm{C}$ before (-) and after (- - ) addition of PAF $10^{-7} \mathrm{M}$. The spectra were measured at an emission wavelength of $450 \mathrm{~nm}$.

of PAF a $15 \mathrm{~nm}$ blue-shift of the Laurdan emission spectrum was observed. The emission maximum in basal conditions (unstimulated PMNs) was at $443 \mathrm{~nm}$, while the emission maximum after stimulation with PAF was at $428 \mathrm{~nm}$. Figure 2 shows Laurdan excitation spectra measured at an emission wavelength of $450 \mathrm{~nm}$. The addition of PAF induced a decrease in intensity at longer wavelengths with respect to basal conditions.

In the presence of L-659,989, no significant changes in the emission or excitation spectra were observed after the addition of PAF.

\section{Discussion}

The fluorescent probe Laurdan has been reported to be incorporated at the hydrophilic-hydrophobic interface of the membrane ${ }^{15}$ with the lauric acid tail anchored in the hydrophobic region of the bilayer. It has been demonstrated that Laurdan displays spectral sensitivity to the polarity of its surrounding, showing a red-shift of the emission in polar solvents, with respect to non-polar solvents. ${ }^{9}$ This behaviour is referred to dipolar relaxation phenomena, that are related to the physical state and the dynamics of the surrounding phospholipid polar head group. ${ }^{9,16}$ In single-phase phospholipid vesicles the dynamics of the surroundings detected by Laurdan is very different in the case of the gel or of the liquid-crystalline phase; the probe shows a marked steady-state emission red-shift in the phospholipid liquid-crystalline phase, with respect to the gel phase. ${ }^{\text {If }}$ If solvent molecules can move during the fluorescence lifetime, the Laurdan excited-state molecular dipole will orient the neighbouring solvent dipoles, and the steady-state Laurdan emission spectrum will be red-shifted. ${ }^{17}$

Our results demonstrate that PAF induced a blue-shift of the emission spectra of Laurdan 
incorporated in the plasma membrane, indicating a decrease in polarity of the environment surrounding the probe. In basal conditions, Laurdan emission reflects the interactions between the probe and the phospholipid polar head groups. The addition of PAF to PMNs decreases the motional freedom of the bilayer polar residues, as demonstrated by the presence of an unrelaxed blue-shift of the spectrum. In previous studies, ${ }^{12,18}$ using the fluorescent probe 1-(4-trimethylammoniumphenyl)6-phenyl-1,3,5-hexatriene (TMA-DPH), we have shown that PAF $10^{-7} \mathrm{M}$ induces an increase in PMNs membrane phospholipid packing and a decrease in membrane microheterogeneity. These physico-chemical changes may cause a reduction of water penetration in the hydrophobic environment surrounding the Laurdan molecules, thus inducing a decrease in polarity. Because these changes in the physico-chemical organization of the membrane can be blocked by the PAF antagonist L-659,989, it seems likely that they are attributed to PAFreceptor interaction and the biochemical events associated with signal transduction.

\section{References}

1. Koltai M, Hosford D, Guinot P, Esanu A, Braquet P. Platelet activating factor (PAF): a review of its effects, antagonists and possible future clinical implications (Part I). Drugs 1991; 42: 9-29.

2. Koltai M, Hosford D, Guinot P, Esanu A, Braqet P. PAF: a review of its effects, antagonists and possible future clinical implications (Part II). Drugs 1991; 42: 174-204

3. Shukla SD. Platelet activating factor receptor and signal transduction mechanisms. FASEB J 1992; 6: 2296-2301.

4. Hwang S-B. Specific receptors of platelet-activating factor, receptor heterogeneity, and signal transduction mechanisms. J Lipid Mediators 1990 2: $123-158$

5. Nakamura M, Honda Z, Izumi T, et al. Molecular cloning and expression of platelet activating factor receptor from human leukocytes. I Biol Chem 1991; 266: 20400-20405

6. Lakowicz JR. Fluorescence polarization. In: Lakowicz JR, ed. Principles of Fluorescence Spectroscopy. New York: Plenum Press, 1983; 709-733.
7. Fiorini R, Gratton E, Curatola G. Effect of cholesterol on membrane microheterogeneity: a study using 1,6-diphenyl-1,3,5-hexatriene fluorescence lifetime distribution. Biochim Biophys Acta 1989; 1006: 198-202.

8. Kantar A, Giorgi PL, Curatola G, Fiorini R. Effect of PAF on erythocyte membrane heterogeneity: a fluorescence study. Agents \& Actions 1991; 32 347-350.

9. Parasassi T, Conti F, Gratton E. Time-resolved fluorescence emission spectra of Laurdan in phospholipid vesicles by multifrequency phase and modulation fluorometry. Cell Biol 1986; 32: 103-108.

10. Ponpipom MM, Hwang S-B, Doebber TW, et al. ( \pm )-trans-2-(3-Methoxy5 - methylsulfonyl - 4 - propoxyphenyl) - 5 - trimethoxyphenyltetrahydrofuran (L-659,989), a novel, potent PAF receptor antagonist. Biochem Biophys Res Commun 1988; 150: 1213-1220.

11. Kantar A, Wilkins G, Swoboda B, et al. Alterations of the respiratory burs of polymorphonuclear leukocytes from diabetic children: a chemiluminescence study. Acta Paediatr Scand 1990; 79: 535-541.

12. Fiorini R, Curatola G, Bertoli E, Giorgi PL, Kantar A. Changes of fluorescence anisotropy in plasma membrane of human polymorphonuclear leukocytes during the respiratory burst phenomenon. FEBS Lett 1990; 273 $122-126$.

13. Fiorini R, Curatola G, Kantar A, Giorgi PL, Gratton E. Use of Laurdan fluorescence in studying plasma membrane organization of polymorphonuclear leukocytes during the respiratory burst. Photochem PhotoBiol 1993; 57 : 438-441.

14. Varveri FS, Mantaka-Marketou AE, Papadopoulos K, Nikokavouras J Chemiluminescence in organized molecular assemblies: chemiluminescence of lucigenin in Lyso-PAF $\left(C_{16}\right)$. J Photochem Photobiol 1992; 66: 113-118.

15. Chong PLG. Effects of hydrostatic pressure on the location of Prodan in lipid bilayers and cellular membranes. Biochemistry 1988; 27: 399-404.

16. Parasassi T, Conti F, Gratton E. Fluorophores in a polar medium: time dependence of emission spectra detected by multifrequency phase and modulation fluorometry. Cell Mol Biol 1986; 32: 99-102.

17. Parasassi T, De Stasio G, D'Ubaldo A, Gratton E. Phase fluctuation in phospholipid membranes revealed by Laurdan fluorescence. Biophys J 1990; 57: 1179-1186.

18. Kantar A, Giorgi PL, Curatola G, Fiorini R. Changes in plasma membrane microheterogeneity of polymorphonuclear leukocytes during the activation of the respiratory burst. In: Jesaitis AJ, Dratz EA, eds. Molecular basis of oxidative damage by leukecytes. Boca Raton: CRC Press, 1992; 243-246.

ACKNOWLEDGEMENTS. Part of this work was performed in the Laboratory for Fluorescence Dynamics (LFD) at the University of Illinois at Urbana-Champaign (UIUC). The LFD is supported jointly by the Division of Research Resources of the NIH and UIUC. We are indebted to Prof. Enrico Gratton for his kind assistance.

\section{Received 6 January 1993} accepted in revised form 15 February 1993 


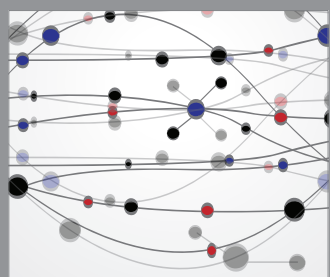

The Scientific World Journal
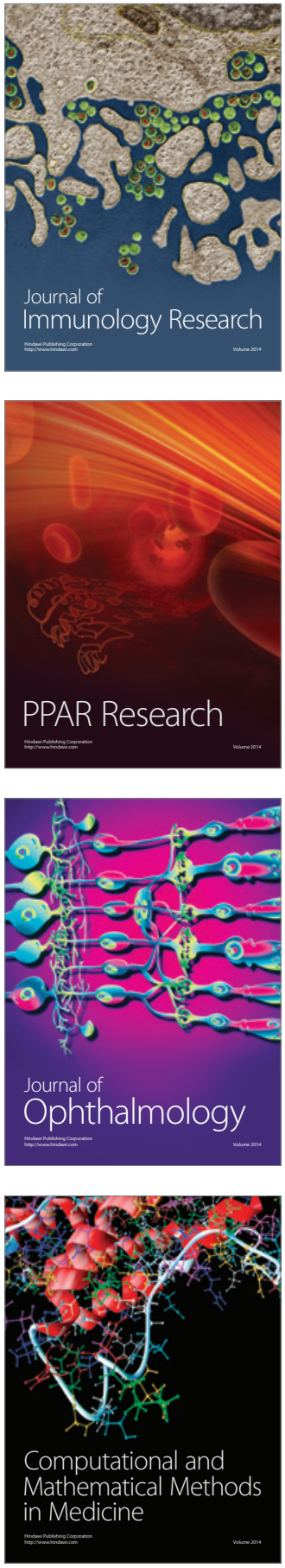

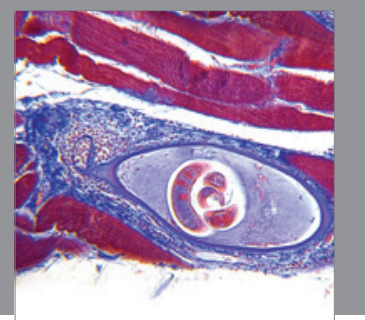

Gastroenterology

Research and Practice
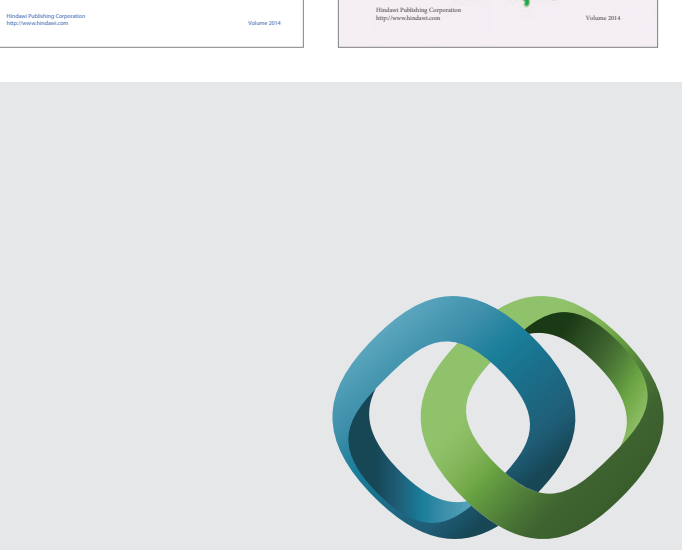

\section{Hindawi}

Submit your manuscripts at

http://www.hindawi.com
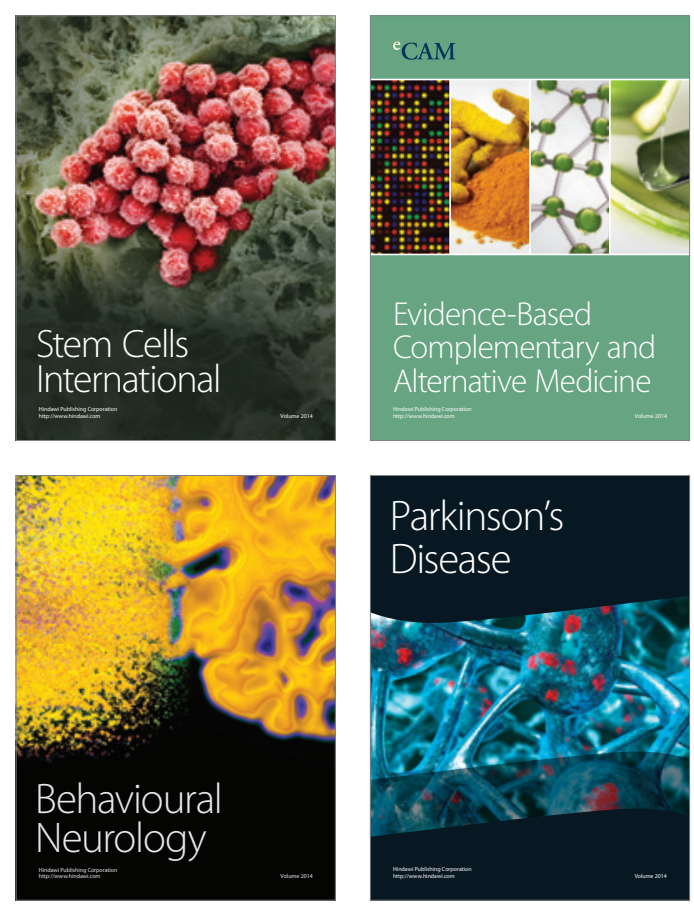

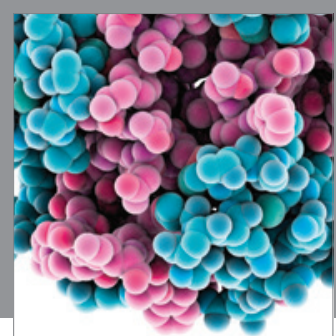

Journal of
Diabetes Research

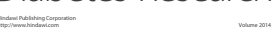

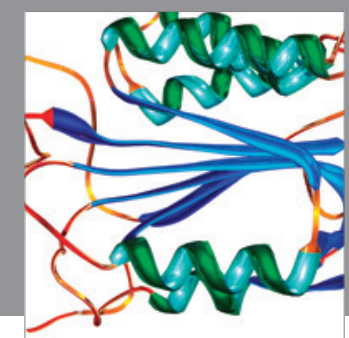

Disease Markers
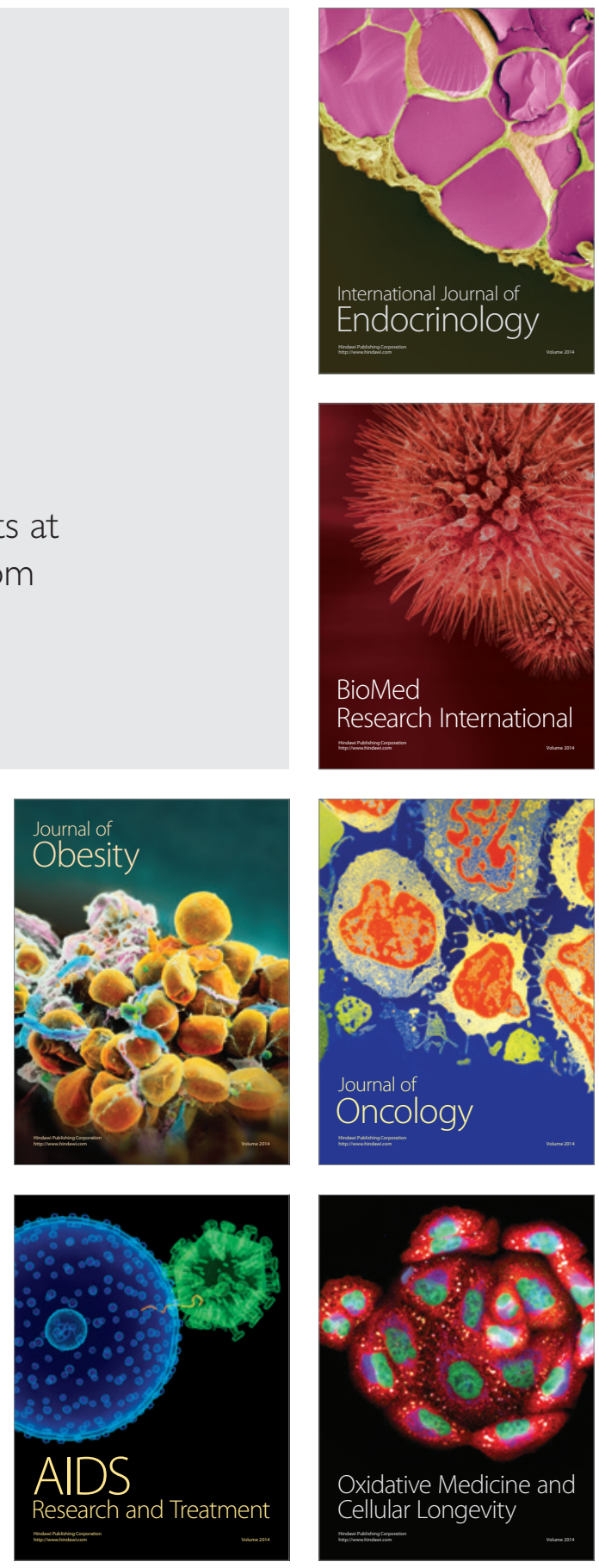